\title{
Simultaneous Identification of Multiple Causal Mutations in Rice
}

\author{
Wei Yan ${ }^{1+}$, Zhufeng Chen ${ }^{2 t}$, Jiawei $\mathrm{Lu}^{2}$, Chunjue $\mathrm{Xu}^{2}$, Gang Xie' ${ }^{2}$, Yiqi $\mathrm{Li}^{2}$, \\ Xing Wang Deng ${ }^{2,3 *}$, Hang $\mathrm{He}^{3 *}$ and Xiaoyan Tang ${ }^{2,4 *}$
}

${ }^{1}$ College of Life Sciences, Capital Normal University, Beijing, China, ${ }^{2}$ Shenzhen Institute of Molecular Crop Design, Shenzhen, China, ${ }^{3}$ College of Life Sciences, Peking University, Beijing, China, ${ }^{4}$ Guangdong Provincial Key Laboratory of Biotechnology for Plant Development, College of Life Sciences, South China Normal University, Guangzhou, China

\section{OPEN ACCESS}

Edited by: Keqiang Wu,

National Taiwan University, Taiwan

Reviewed by:

Mingsheng Chen,

Institute of Genetics and Developmental Biology (CAS), China

Chengcai Chu,

Institute of Genetics and

Developmental Biology (CAS), China

*Correspondence:

Xing Wang Deng

deng@pku.edu.cn

Hang $\mathrm{He}$

hehang@pku.edu.cn

Xiaoyan Tang

txy@frontier-ag.com

tThese authors have contributed equally to this article.

Specialty section: This article was submitted to Plant Genetics and Genomics,

a section of the journal

Frontiers in Plant Science

Received: 06 November 2016 Accepted: 23 December 2016 Published: 17 January 2017

Citation:

Yan W, Chen Z, Lu J, Xu C, Xie G,

Li Y, Deng XW, He H and Tang $X$ (2017) Simultaneous Identification of Multiple Causal Mutations in Rice.

Front. Plant Sci. 7:2055.

doi: 10.3389/fpls.2016.02055
Next-generation sequencing technologies (NGST) are being used to discover causal mutations in ethyl methanesulfonate (EMS)-mutagenized plant populations. However, the published protocols often deliver too many candidate sites and sometimes fail to find the mutant gene of interest. Accurate identification of the causal mutation from massive background polymorphisms and sequencing deficiencies remains challenging. Here we describe a NGST-based method, named SIMM, that can simultaneously identify the causal mutations in multiple independent mutants. Multiple rice mutants derived from the same parental line were back-crossed, and for each mutant, the derived F2 individuals of the recessive mutant phenotype were pooled and sequenced. The resulting sequences were aligned to the Nipponbare reference genome, and single nucleotide polymorphisms (SNPs) were subsequently compared among the mutants. Allele index (Al) and Euclidean distance (ED) were incorporated into the analysis to reduce noises caused by background polymorphisms and re-sequencing errors. Corrections of sequence bias against GC- and AT-rich sequences in the candidate region were conducted when necessary. Using this method, we successfully identified seven new mutant alleles from Huanghuazhan $(\mathrm{HHZ})$, an elite indica rice cultivar in China. All mutant alleles were validated by phenotype association assay. A pipeline based on Perl scripts for SIMM is publicly available at https://sourceforge.net/projects/simm/.

Keywords: SIMM, next-generation sequencing technology, single nucleotide polymorphism, SNP index, allele index, Euclidean distance, sequence correction

\section{INTRODUCTION}

Forward genetics based on EMS mutagenesis and cloning of mutant genes are of fundamental importance to the understanding of plant gene functions. Traditional map-based cloning approach has been widely used for cloning of mutant plant genes. It involves construction of mapping population(s) by crossing the mutant plant with wild-type plant of extensive genomic polymorphisms (Lukowitz et al., 2000). DNA sequence polymorphisms, such as simple sequence repeats, SNPs, and short insertions or deletions, are often used as markers for determination of parental chromosomal locations in the mapping population (Peters et al., 2003). By identifying markers that are tightly associated with the mutant phenotype, chromosomal region harboring

Abbreviations: AI, allele index; ED, Euclidean distance; EMS, ethyl methanesulfonate; HHZ, Huanghuazhan; HRM; high resolution melting; NGST, next-generation sequencing technology; SIMM, simultaneous identification of multiple mutations; SI, SNP index; SNP, single nucleotide polymorphism. 
the causal mutation can be identified. The mutant gene is further defined by sequencing the chromosomal region and functional complementation (Lukowitz et al., 2000; Jander et al., 2002). Despite the wide application, map-based cloning is laborintensive and time-consuming. In addition, it is not suitable for cloning of mutant genes with ambiguous phenotypes in the mapping population.

The rapid development of NGST renders the sequencing of plant genomes routine. Consequently, several NGST-based methods have been developed for cloning of the mutant genes, including SHOREmap (Schneeberger et al., 2009), NGM (Austin et al., 2011), MutMap (Abe et al., 2012), MutMap+ (Fekih et al., 2013), and NIKS (Nordstrom et al., 2013).

Like map-based cloning, SHOREmap and NGM both involve crossing of the mutant with a remotely related wild-type plant; the derived F2 plants of the mutant phenotype are bulksequenced. Both methods have to deal with the numerous background polymorphisms between the two crossing accessions, which render it difficult to determine causal mutations. Thus far, SHOREmap, NGM, and other related methods have been successful only for cloning of mutant genes in Arabidopsis (Schneeberger et al., 2009; Austin et al., 2011; Uchida et al., 2011).

MutMap (Abe et al., 2012) takes a different approach by back-crossing the mutant with the wild-type parent, and 2030 derived F2 individuals of the mutant phenotype are bulksequenced. MutMap requires sequencing and construction of a pseudo-genome for the wild-type parent prior to sequence alignment with the bulk sequence of the mutant plant. The pseudo-genome is generated by aligning the genome sequences of the wild-type parent with an assembled reference genome and then replacing the SNPs. Because MutMap deals with only the SNPs incorporated by mutagenesis, many fewer sequence polymorphisms are encountered in sequence alignment. Methods related to MutMap have been successfully used for cloning of mutant genes in Arabidopsis and rice (Abe et al., 2012; Zhu et al., 2012; Allen et al., 2013; Takagi et al., 2013).

Unlike MutMap, MutMap+ (Fekih et al., 2013) does not require backcross of the mutant with the wild-type parent. Instead, a single M2 heterozygous plant is selfed, and the derived M3 plants of the mutant and wild-type phenotypes are bulksequenced and then respectively aligned to the pseudo-genome of the wild-type parent. MutMap + is particularly useful for cloning of mutant genes causing lethal or infertile phenotype that are not amenable for crossing.

NIKS (Nordstrom et al., 2013) is a reference-free algorithm that can be used for discovery of causal mutations even in plants that do not have an assembled reference genome sequence. It relies on the analysis of $k$-mers, which are defined as subsequences of length $k$ of a sequencing read. The plants to be bulksequenced are generated as described for MutMap or MutMap ${ }^{+}$. NIKS starts by assessing the frequency of each $k$-mer within the bulk sequencing data. $k$-mers of identical sequences are saved as seeds for further comparison with the sequence of the wild-type parent, while $k$-mers of polymorphic sequences are discarded. In theory, NIKS can be applied to identify mutant genes that are caused not only by SNPs but also by small indels and large deletions.
The aforementioned NGST-based methods significantly accelerate the cloning of mutant genes in plants. However, since these methods depend on the comparison of the mutant bulks with wild-type bulks, they frequently produce dozens of candidate sites and sometimes fail to identify causal mutations (Nordstrom et al., 2013). There is a clear need for a method that can better resolve causal mutations. Here we report a method named SIMM for precise identification of multiple causal mutations in rice. SIMM does not need the genome sequence of the wild-type parent as reference. It can resolve candidate mutations to one or a few SNPs. Applications on seven published rice mutants and seven HHZ mutants indicated that SIMM is a proficient method which can simultaneously resolve the causal genes in multiple mutants.

\section{MATERIALS AND METHODS}

\section{EMS Mutagenesis and Mutant Screening}

Sixty kilograms of $\mathrm{HHZ}$ seeds were soaked in water for $22 \mathrm{~h}$ and then drained. The drained seeds were incubated with $0.7 \%$ EMS solution at $28^{\circ} \mathrm{C}$ for $12 \mathrm{~h}$ and stirred every $30 \mathrm{~min}$. The seeds were then washed five times with water, each time for $10 \mathrm{~min}$, and then washed with running water for $1.5 \mathrm{~h}$. The seed germination rate was determined to be $\sim 75 \%$. Mutagenized seeds were cultivated in field, and seeds from $\sim 1,000$ plants were pooled. Approximately 40,000 grains from each pool were planted, and mutants of various abnormal phenotypes were screened from 50 pools of the mutant library.

\section{Genetic Analysis of Mutants and Construction of Sequencing Population}

Fertile mutants of abnormal phenotype were allowed to set seeds. For each mutant, 40 seeds were planted for phenotype confirmation. One plant with an inheritable phenotype was chosen to back-cross with wild-type HHZ, and the derived F1 plants were further selfed to produce F2 seeds. Sterile mutants were directly crossed with wild-type HHZ to produce F1, which then selfed to produce F2 seeds. Phenotype segregation ratios were evaluated with 160 F2 plants, and those displaying 3:1 segregation of wild-type to mutant plants were regarded as recessive mutants. Thirty F2 individuals of the mutant phenotype were selected for DNA extraction.

\section{DNA Extraction and Sequencing}

Total DNA was extracted using DNeasy Plant Mini Kit (Qiagen) according to the manufacturer's protocol and quantified using a Nanodrop ${ }^{\mathrm{TM}} 2000$ spectrophotometer (Thermo Scientific). Equal amounts of DNA from each plant were pooled, and $5 \mu \mathrm{g}$ of the pooled DNA was used for preparation of a sequencing library of average insert size 200-500bp, according to the protocol for the Paired-End DNA Sample Prep Kit (Illumina). The library was sequenced to $25 \sim 40 \mathrm{x}$ of genome coverage with the Illumina Hiseq 2000 platform. 


\section{Correction of Sites of Low Coverage Depth or Low Quality}

A mutant with defined candidate regions but no identifiable causal mutations was subjected to site correction of quality and coverage depth based on the GC content. Correction was applied only to those sites with base quality $<20$ or depth between 5 and 15. Site correction was performed according to following rules: 1 , The average depth $\bar{D}_{\mathrm{b} / \mathrm{c}}$ for each GC content group is calculated as $\overline{\mathrm{D}}_{\mathrm{b} / \mathrm{c}}=\sum_{\mathrm{i}=1}^{\mathrm{N}} D_{\mathrm{i}} / \mathrm{N}$, where $N$ is the number of non-overlapping $200 \mathrm{bp}$ bins randomly selected from the background regions $(b)$ and candidate regions $(c)$. The maximal base depth is limited to 300 to minify the influence of repetitive regions on calculation of the average depth. 2, The average quality $\overline{\mathrm{Q}}_{\mathrm{b} / \mathrm{c}}$ for each GC content group is calculated as $\bar{Q}_{\mathrm{b} / \mathrm{c}}=\sum_{\mathrm{i}=1}^{\mathrm{N}} \mathrm{Q}_{\mathrm{i}} / \mathrm{N}$. 3, The depth coefficient (d) and quality coefficient $(q)$ are respectively calculated as $d=\max \left(\overline{\mathrm{D}}_{\mathrm{c}}, \bar{D}_{\mathrm{b}}\right) /\left(\mathrm{D}_{\mathrm{i}} / 200\right)$ and $q=\max \left(\overline{\mathrm{Q}}_{\mathrm{c}}, \overline{\mathrm{Q}}_{\mathrm{b}}\right) /\left(\mathrm{Q}_{\mathrm{i}} / 200\right)$. 4 , The quality coefficient or depth coefficient was multiplied with the actual base quality or actual base depth in the same GC content group to get the corrected quality or corrected depth for each site. The corrected values were then used for SNP calling and calculation of SNP index and ED values.

\section{High Resolution Melting Analysis}

Total DNA was extracted from rice leaves using a modified CTAB method (Allen et al., 2006) and diluted to $100 \mathrm{ng} / \mu \mathrm{l}$. PCR reactions contained final concentrations of $0.05 \mathrm{U} / \mu \mathrm{l}$ Taq DNA polymerase (Takara), 1x PCR buffer, $25 \mu \mathrm{M}$ dNTP each, $0.1 \mu \mathrm{M}$ each primer, $5 \mathrm{ng} / \mu \mathrm{l}$ DNA template, $0.2 \mathrm{x}$ EvaGreen (Biotium), and were made up to $10 \mu \mathrm{l}$ with water. Reactions were cycled on a thermal cycler $\left(94^{\circ} \mathrm{C} 3 \mathrm{~min}, 40\right.$ cycles of $\left[94^{\circ} \mathrm{C} 30 \mathrm{~s}, 58^{\circ} \mathrm{C} 30 \mathrm{~s}, 72^{\circ} \mathrm{C} 10 \mathrm{~s}\right], 72^{\circ} \mathrm{C} 5 \mathrm{~min}, 95^{\circ} \mathrm{C} 1 \mathrm{~min}$, $25^{\circ} \mathrm{C} 1 \mathrm{~min}$ ) and internal temperature calibrators were added into the amplification products. HRM data were collected on a LightScanner (Idaho Technology) $\left(53 \sim 98^{\circ} \mathrm{C}\right.$, hold at $50^{\circ} \mathrm{C}$ ) and analyzed with the LightScanner Call-IT software (Idaho Technology).

\section{RESULTS}

\section{Principle of SIMM}

Simultaneous identification of multiple mutations was primarily designed to identify casual mutations generated by EMS mutagenesis by simultaneous analysis of multiple mutants derived from the same parental plant, without requiring a wildtype parental genome sequence as reference (Figure 1). Each discrete mutant was back-crossed with the wild-type parent, and 20-30 F2 individuals of the recessive mutant phenotype were pooled and sequenced to $>20 \mathrm{x}$ of the genome size using the Illumina Hiseq 2000 platform.

The raw short reads were first cleaned of adapter sequences, low quality reads (average Phred quality $<20$ ), and reads containing $>10 \%$ Ns using Cutadapt (Martin, 2011). Clean reads

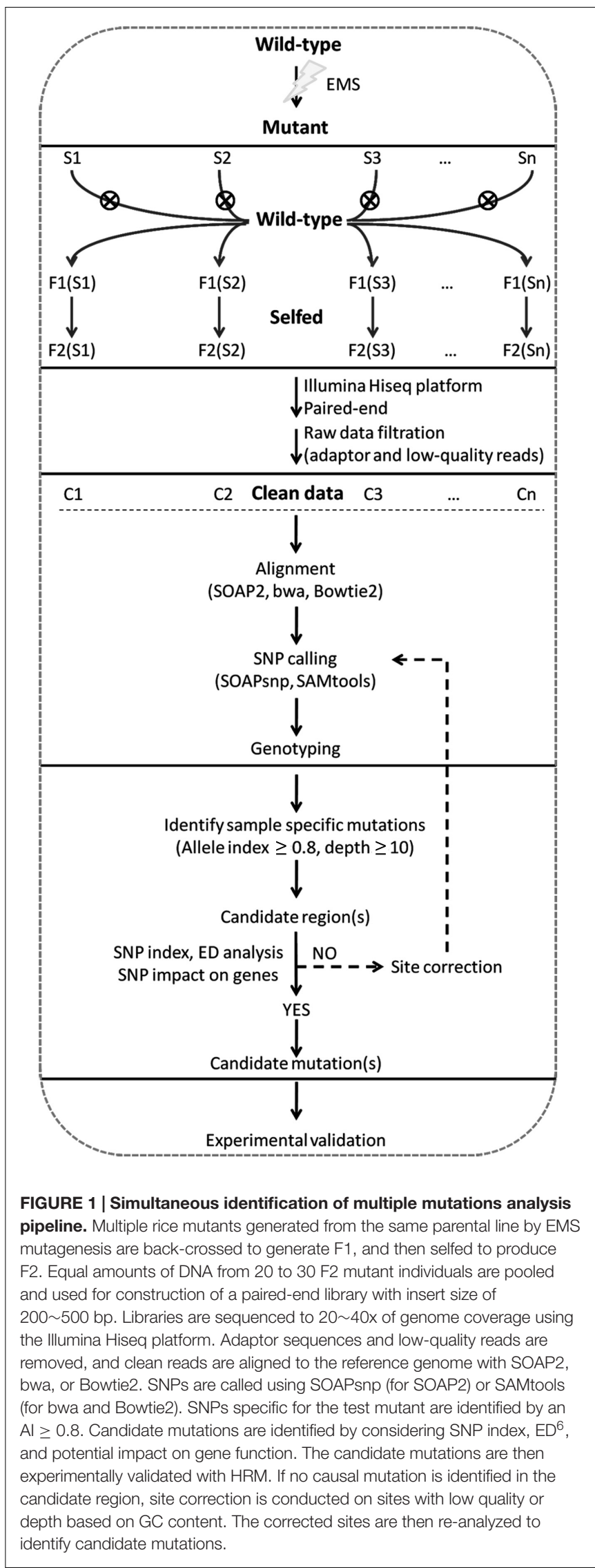


for each sample were then aligned to the Nipponbare reference genome (Release 7 of the MSU Rice Genome Annotation Project) ${ }^{1}$ using software SOAP2 (Li et al., 2009b), bwa (Li and Durbin, 2009), or Bowtie2 (Langmead and Salzberg, 2012). Reads that aligned to two or more chromosomal positions were discarded due to the uncertainty of their genomic origin. Only uniquely mapped reads were retained for SNP calling using SOAPsnp (Li et al., 2009a) (for SOAP2) or SAMtools (Li H. et al., 2009) (for bwa and Bowtie2). Total SNPs between each mutant bulk and Nipponbare were identified and further compared among the mutants to identify SNPs specific for each discrete mutant strain. SNPs with $<5$ supporting reads in the mutant were discarded, as those may have resulted from sequencing errors or insufficient clusters.

MutMap and MutMap+ both compare EMS mutants with the wild-type parental line (Abe et al., 2012; Fekih et al., 2013). However, sequencing deficiencies such as sequencing errors and insufficient clusters often introduce errors into this pair-wise comparison. To overcome this problem, we introduced an AI to assess SNPs supporting wild-type alleles in background mutants (i.e., all mutant strains except the test mutant). AI was calculated as the number of reads supporting the wild-type genotype divided by the total number of reads covering the site in the background mutants. A SNP was regarded as test strain-specific when AI of this site was $\geq 0.8$, allowing $20 \%$ sequencing errors in background mutants. Test strain-specific SNPs with read depth $\geq 10$ were further assessed with SI, which was calculated as the number of mutant reads divided by the number of total reads in the test mutant. SNP responsible for the mutant phenotype should have a $\mathrm{SI}=1$, but due to the potential of sequencing errors, we lowered the cutoff of SI to 0.8 . Those sites with $\mathrm{SI} \geq 0.8$ were all retained as candidates.

Although SI was a good indicator of candidacy, there were often too many sites when using $\mathrm{SI} \geq 0.8$ as threshold. To further refine candidate SNPs, ED analysis (Hill et al., 2013) was employed, which considered the SNP index in the test strain and reference mutants. $E D$ value was calculated as $E D=\sqrt{2 \times\left(S I_{t}-S I_{b c}\right)^{2}}$, where $S I_{t}$ stands for the SI of mutation allele in the test strain, whereas $S I_{b c}$ stands for the SI of the same mutation allele in the background mutants. Since ED value ranged from 0 to $\sqrt{2}$, it was raised to power $6\left(\mathrm{ED}^{6}\right)$, which was sufficient to enlarge the differences between causal mutations and closely linked mutations, and to signify candidate regions. Local linear regression curve (Loess fit) (Jacoby, 2000) was appended to show the distribution of SI and $\mathrm{ED}^{6}$ values along the chromosomes. Candidate regions harboring the causal mutation are expected to show a cluster of SNPs with high SI and $\mathrm{ED}^{6}$ values. Finally, the impacts of SNPs on gene function were evaluated. Mutations at intron splicing sites or non-synonymous mutations were ranked highest, followed by mutations in the promoter region, intron, and intergenic region.

If the aforementioned protocol correctly identifies the candidate region but fails to identify the causal mutation, we applied a correction of sites in the candidate region based on GC content. SNPs filtered out by quality and/or depth criteria

${ }^{1}$ http://rice.plantbiology.msu.edu/ were corrected according to the quality coefficient and/or quality coefficient, and then subjected to the SIMM pipeline. Ultimately, the candidate mutations were experimentally validated using phenotype association assay.

\section{Application of SIMM on Seven Previously Analyzed Rice Mutants}

To evaluate the performance of SIMM, we first analyzed the bulksequencing data of seven rice mutants released by Abe et al. (2012). These data were analyzed using both MutMap (Abe et al., 2012) and NIKS (Nordstrom et al., 2013). MutMap revealed $101 \sim 238$ candidate sites with $\mathrm{SI} \geq 0.9$. Among them, $4 \sim 24$ were of CG to TA transitions common to EMS mutagenesis. NIKS revealed $7 \sim 21$ candidate mutations of CG to TA transitions, but the number of other mutation types was not reported. SIMM revealed $6 \sim 32$ candidate sites for each mutant with $\mathrm{SI} \geq 0.8$, of which $4 \sim 17$ were CG to TA mutations (Table 1). When ED analysis was applied, candidate sites for each mutant were reduced to $3 \sim 15$ (Table $\mathbf{1}$ ).

The candidate sites were further evaluated of functional impacts on the genes, resulting in a final list of 12 candidate genes for these mutants, six of which were missed when using MutMap, and two were missed when using NIKS (Table 1). Candidate genes revealed by SIMM and NIKS were mostly identical except for the mutants Hit1917-sd and Hit0813pl2. The candidate site identified by MutMap in mutant Hit1917-sd was identified neither by NIKS nor by SIMM (Abe et al., 2012; Nordstrom et al., 2013). SIMM identified two candidate sites for Hit1917-sd; one of them was also identified by NIKS, but the other one at the intron splicing site of LOC_Os12g37870 was missed by NIKS (Table 1). MutMap did not detect either of the two sites (Table 1). LOC_Os12g37870 is orthologous to AT1G09430 encoding an ATP-citrate lyase. Arabidopsis plants expressing AT1G09430 antisense RNA exhibit dwarfism (Fatland et al., 2005), which was consistent with the dwarf phenotype of Hit1917-sd (Abe et al., 2012). Based on these observations, we propose LOC_Os12g37870 as the potential mutant gene for Hit1917-sd. For the pale green mutant Hit0813-pl2, NIKS detected an SNP in the intron of LOC_Os01g72800, a chloroplast SRP receptor. SIMM discovered this SNP and three other mutation sites, one in the intron of LOC_Os01g74460, and two in intergenic regions. Significantly, the orthologous gene of LOC_Os01g74460 in Arabidopsis (AT2G28390) encodes a chloroplast-located protein. We consider both LOC_Os01g72800 and LOC_Os01g74460 to be prime candidates for the pale green phenotype of mutant Hit0813-pl2. These results indicated that SIMM has higher sensitivity and specificity than the other two methods in localizing the candidate genes.

\section{Identification of HHZ Mutant Genes Using SIMM}

We obtained seven HHZ mutants that are controlled by single recessive genes (Figure 2). We first used the MutMap method to identify the causal mutations, so the wild-type HHZ was sequenced to $\sim 10 \mathrm{x}$ of genome coverage. The bulked sequences 
TABLE 1 | Comparison of results from MutMap, NIKS and SIMM analyses on seven published mutants.

\begin{tabular}{|c|c|c|c|c|c|c|c|c|}
\hline Sample & MutMap' & $\mathrm{NIKS}^{2}$ & SIMM $^{3}$ & $\mathrm{ED}^{4}$ & Chr & Locus & Genotype/aa & Candidate gene \\
\hline Hit0746-sd & $121(13)$ & 16 & $8(8)$ & 3 & Chr8 & $27,272,776$ & $G$ to $A(D$ to $N)$ & LOC_Os08g43120 b,c \\
\hline Hit5243-sm & $229(21)$ & 19 & $32(14)$ & 15 & Chr8 & $11,552,593$ & $\mathrm{C}$ to $\mathrm{T}(\mathrm{R}$ to $\mathrm{C})$ & LOC_Os08g19310a,b,c \\
\hline Hit5500-sd & $131(9)$ & 12 & $10(4)$ & 8 & Chr9 & $20,062,177$ & $\mathrm{~T}$ to $\mathrm{A}$ ( $\mathrm{L}$ to stop) & LOC_Os09g33980a,b,c \\
\hline \multirow[t]{2}{*}{ Hit1917-pl1 } & $101(24)$ & 21 & $19(18)$ & 7 & Chr10 & $22,484,681$ & $\mathrm{C}$ to $\mathrm{T}(\mathrm{L}$ to $\mathrm{F})$ & LOC_Os10g41780a,b,c \\
\hline & & & & & & $22,705,386$ & $C$ to $T$ (A to $V$ ) & LOC_Os10g42196 b,c \\
\hline \multirow[t]{2}{*}{ Hit1917-sd } & $139(10)$ & 7 & $12(12)$ & 9 & Chr12 & 23,278,301 & G to $A$ (splicing site) & LOC_Os12g37870 \\
\hline & & & & & & $23,285,822$ & $\mathrm{G}$ to $\mathrm{A}(\mathrm{S}$ to $\mathrm{N})$ & LOC_Os12g37890b,c \\
\hline \multirow[t]{3}{*}{ Hit5814-sd } & $121(4)$ & 10 & $6(4)$ & 3 & Chr4 & $23,555,382$ & A to $T$ (R to stop) & LOC_Os04g39560a,b,c \\
\hline & & & & & & $24,669,920$ & $\mathrm{C}$ to $\mathrm{T}(\mathrm{L}$ to $\mathrm{F})$ & LOC_Os04g41580a,b,c \\
\hline & & & & & & $25,537,772$ & $\mathrm{C}$ to $\mathrm{T}$ (R to $\mathrm{C}$ ) & LOC_Os04g43140a,b,c \\
\hline \multirow[t]{4}{*}{ Hit0813-pl2 } & $238(10)$ & 20 & $6(6)$ & 4 & Chr1 & $42,235,284$ & G to A (intron) & LOC_Os01g72800 b,c \\
\hline & & & & & & $42,282,527$ & $\mathrm{C}$ to $\mathrm{T}$ (intergenic) & - \\
\hline & & & & & & $42,843,286$ & $\mathrm{C}$ to $\mathrm{T}$ (intergenic) & - \\
\hline & & & & & & $43,121,603$ & G to $A$ (intron) & LOC_Os01g74460 \\
\hline
\end{tabular}

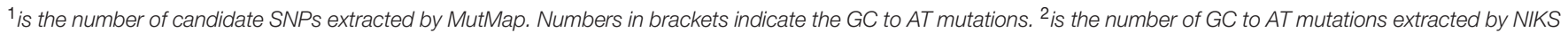

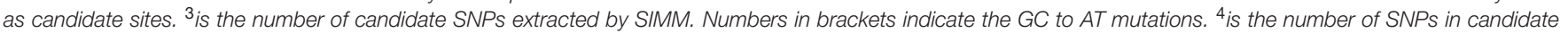

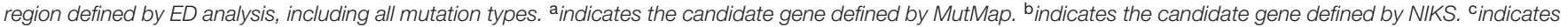
the candidate gene defined SIMM.

of each mutant and wild-type HHZ were respectively aligned to the Nipponbare reference genome, which generated a total of 1.21-1.61 $\times 10^{6}$ SNPs for the mutants and $1.00 \times 10^{6}$ SNPs for wild-type HHZ (Table 2, \#SNPs ${ }^{1}$ ). We then compared the SNPs of each mutant against wild-type HHZ to identify the SNPs specific for each mutant, resulting in a total of 5,108 9,230 specific SNPs (Table 2, \#SNPs ${ }^{2}$ ), of which, 955 1,546 had SI $\geq 0.8$ (Table 2 , \#SNPs ${ }^{2}$, numbers in bracket). These SNPs were presumably the candidate mutations. However, it was not possible to pinpoint causal mutations from so many candidates.

We then took the SIMM approach by comparing the SNPs of one mutant strain against the SNPs of all other mutant stains, which generated 330 1,109 SNPs specific for each mutant (Table 2, \#SNPs ${ }^{3}$ ), except for the mutant $\mathrm{H}-212$ which was contaminated by fungal DNA, and HT5763 that contains large unique genomic regions absent in other mutant strains. SNP index analysis identified $7 \sim 233$ candidate SNPs with $S I \geq 0.8$ and read depth $\geq 10$ (Table 2 , $\# \mathrm{SNPs}^{3}$, numbers in bracket), which were much smaller than those identified with MutMap (Table 2, $\# \mathrm{SNPs}^{2}$, numbers in bracket). ED analysis further refined the candidate regions and narrowed down to 4-20 candidate sites for these mutants (Table 2, $\# \mathrm{SNPs}^{4}$ ). Our initial functional assessment pinpointed one causal gene for each of these mutants, except H-212 (Table 2).

Mutant H-224 exhibited abnormal spikelet and seed morphology (Figures 2C,D). The palea and lemma were not tightly interlocked, resulting in seeds with open beaks and hulls. A large portion of the spikelets and seeds exhibited brownish palea and/or lemma with poorly filled grain inside. SNP index analysis indicated three putative candidate regions (Figure 3A). However, ED analysis highlighted a region on chromosome 3 containing 9 SNPs of high scores (Figure 3A). Only the SNP (Chr3: 24,417,520) located in LOC_Os03g43670 (OsCR4) causes amino acid substitution from Gly (GGC) to Asp (GAC) (Table 2).
We could not find OsCR4 mutants in rice, but RNAi inhibition of OsCR4 expression led to abnormal spikelets and seeds with phenotypes similar to what we observed in H-224 mutant (Pu et al., 2012). The less significant peak on chromosome 10 probably represents a different phenotype in the sequenced F2 mutants.

Mutant HT5763 was a male sterile mutant (Figures 2E,F). Both SNP index and ED analyses identified multiple candidate regions harboring SNPs of high scores (Figure 3B). Regions located on chromosome 2, 3, and 9 have high SNP densities, giving rise to a much larger number of specific SNPs in HT5763 than in other mutants (Table 2). These regions are likely heterologous chromosomal fragments that were still segregating in the EMS-treated seed population. The left end of chromosome 11 has two SNPs of high scores; one is a synonymous mutation, and the other one is in an intergenic region. Another candidate region on chromosome 4 carries a SNP (Chr4: 23,511,644) in LOC_Os04g39470 (OsMYB80) causing an amino acid substitution from Glu (GAG) to Lys (AAG) (Table 2). OsMYB80 is highly homologous to the Arabidopsis AtMYB80 (also called AtMYB103) transcription factor (Higginson et al., 2003). AtMYB80 is required for pollen development in Arabidopsis (Phan et al., 2011). Mutation of this gene in Arabidopsis leads to male sterility. Complementation of the Arabidopsis myb80 mutant with the rice OsMYB80 gene restores male fertility (Phan et al., 2012), suggesting the orthologous relationship between OsMYB80 and AtMYB80. However, the function of OsMYB80 in rice has not been confirmed by mutational analysis. Mutant HT5763 is male sterile with no pollen grains, indicating that OsMYB80 may regulate pollen development in rice. Mutant HT5763 provides a tool for functional study of OsMYB80 in rice.

H-190, H-174, and HM2-S61 were three other male sterile mutants (Figures 2G-L). Both SNP index analysis and ED analysis identified only one prominent candidate region for 

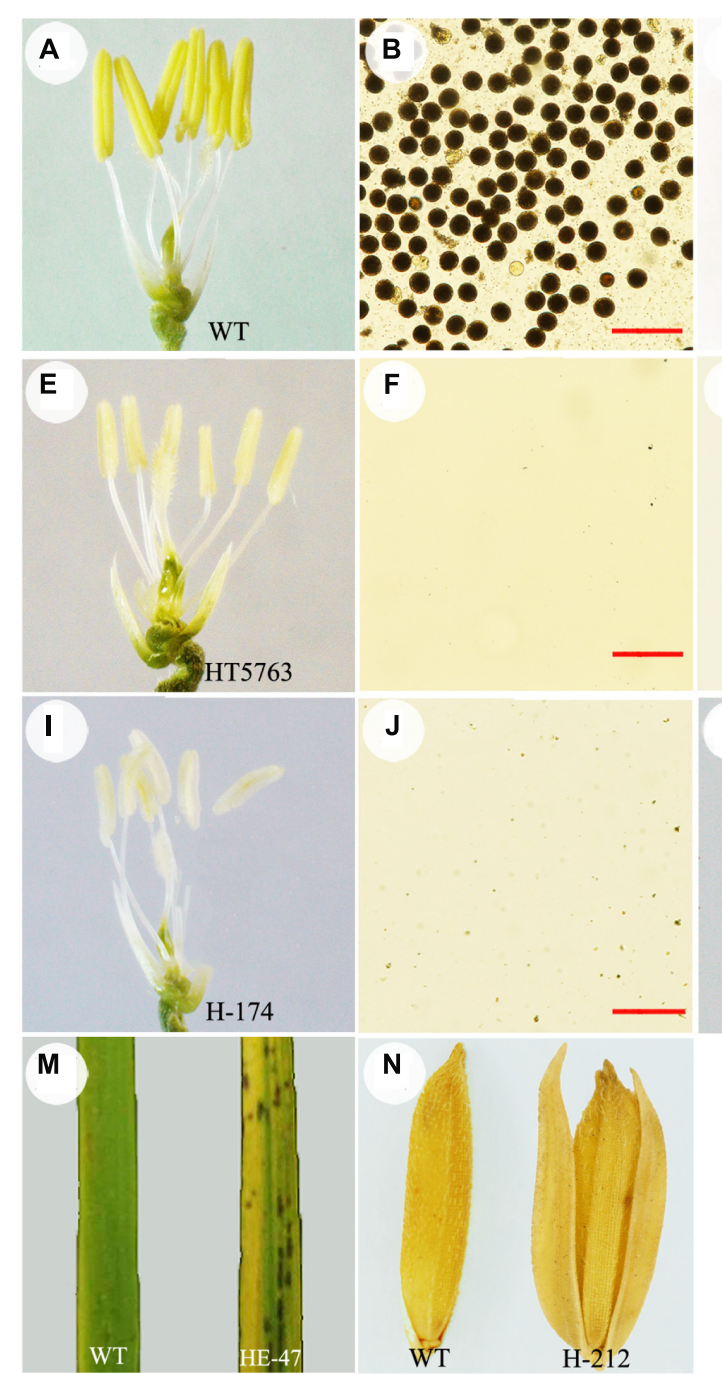

$\mathbf{F}$

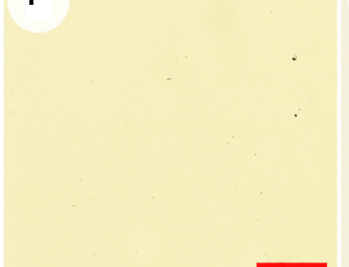

$\mathbf{J}$

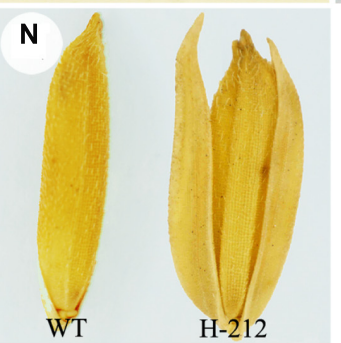

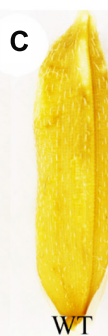
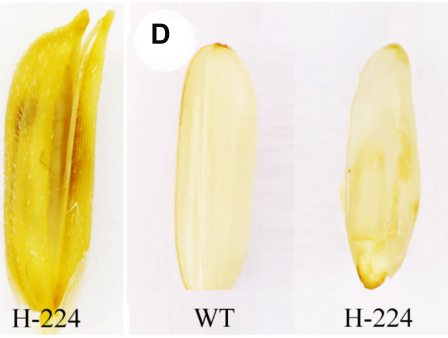

G

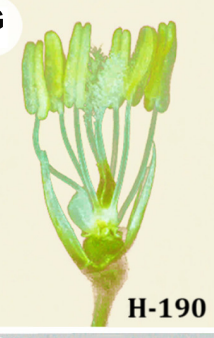

H

FIGURE 2 | Phenotype of seven HHZ mutants. (A,B) wild-type HHZ; (C,D) H-224; (E,F) HT5763; (G,H) H-190; (I,J) H-174; (K,L) HM2-S61; (M) HE-47; (N) $\mathbf{H}$-212. Palea and lemma were removed to show the anthers for the male sterile mutants. Pollen grains in $\mathbf{( B , F , H , J , L )}$ were released from the anthers and stained with $1 \% \mathrm{I}_{2}-\mathrm{KI}$ solution. Seed, husked seed, and leaf from wild-type $\mathrm{HHZ}(\mathrm{WT})$ are shown in (C,D,M,N) as references. Bar $=100 \mu \mathrm{m}$.

these mutants. The candidate regions for $\mathrm{H}-190$ and $\mathrm{H}-174$ are at the end of chromosome 3 that harbor 7 and 13 candidate SNPs, respectively (Figures 3C,D). H-190 has a SNP (Chr3: $33,373,677)$ in the 15th exon of LOC_Os03g58600 (MEL1) that causes amino acid substitution from Tyr (TAC) to Asp (GAC) (Table 2). H-174 has a SNP (Chr3: 33,372,744) in the 20th exon of the same gene that causes amino acid substitution from Glu (GAG) to Lys (AAG) (Table 2). MEL1 encodes an ARGONAUTE protein regulating male and female germ cell development, and mutations of this gene was reported to induce male sterility in rice (Nonomura et al., 2007). The candidate region for HM2S61 is on chromosome 7 that harbors 4 SNPs (Figure 3E). One of these SNPs (Chr7: 19,329,232) located in LOC_Os07g32480 (Brk1) causes amino acid substitution from Gly (GGT) to Ser (AGT) (Table 2). Brk1 encodes a spindle checkpoint kinase acting during rice meiosis. A mutant allele of this gene was reported to be male sterile (Wang et al., 2012), which is consistent with our observation.

Mutant HE-47 has numerous red spots on leaves (Figure 2M). Three regions harboring multiple SNPs of high scores were identified for this mutant. The regions on chromosomes 2 and 10 were dismissed because SNPs in these regions were densely distributed (Figure 3F), which may represent heterologous genomic regions that are still segregating in the EMS-treated seeds. One region at the end of chromosome 3 contains 4 SNPs of high $\mathrm{ED}^{6}$ scores, one of which $(\mathrm{Chr} 3: 3,206,988)$ located in LOC_Os03g06410 (OsEDR1) causes amino acid substitution from Ser (TCA) to Pro (CCA) (Table 2). OsEDR1 encodes a MAPK kinase kinase with a function in plant defense signaling. A TDNA insertion allele of this gene shows spontaneous lesions on rice leaves (Shen et al., 2011), the same phenotype as we observed in mutant HE-47. 


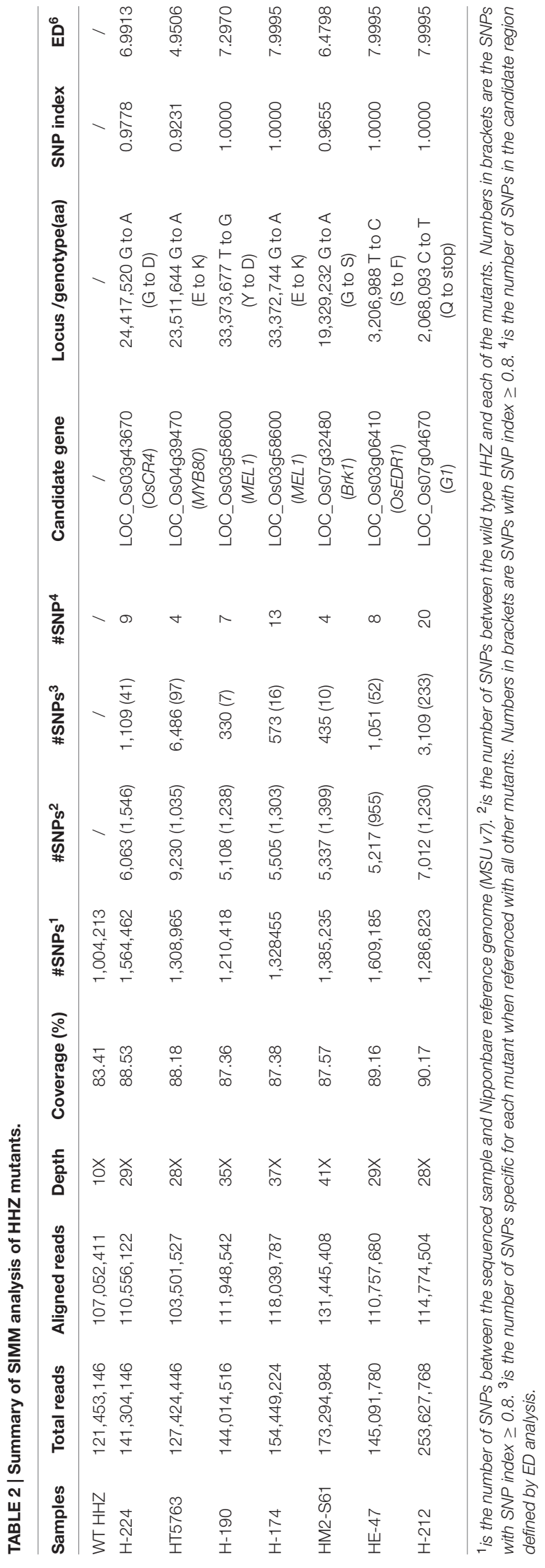

\section{Applying Sequence Correction to Identify the Causal Mutation in H-212}

Mutant $\mathrm{H}-212$ exhibited longer sterile lemmas compared with wild-type HHZ (Figure 2N). Notably, this mutant showed abnormal re-sequencing data with significantly lower aligned ratios to the reference genome, and the base contents of reads were significantly different from what in other sequenced mutants. We randomly chose 100 unmapped reads and blasted in NCBI, which showed that most of these reads originated from Fusarium. To make use of the sequence data, sequences highly similar to the Fusarium sequences were discarded. Then $10 \mathrm{bp}$ at the $5^{\prime}$ end and $5 \mathrm{bp}$ at the $3^{\prime}$ end of both paired-end reads were trimmed from the retained reads, followed by filtration with average Phred quality $\geq 20$. The remaining reads were then searched for the causal mutations.

Three candidate regions were identified on chromosomes 2 , 7, and 10, respectively (Figure 3G). Regions on chromosomes 2 and 10 were dismissed due to high SNP density. Twenty SNPs were identified in the candidate region on chromosome 7, two of which caused non-synonymous mutations. However, all these mutations showed segregation when a large number of F2 individuals were analyzed in phenotype association assays, indicating none being the causal mutation.

Sequences generated by the Illumina Hiseq platforms tend to have low read coverage in GC-rich or AT-rich regions (Benjamini and Speed, 2012). We also found low mapping quality for these regions (Figures $4 \mathbf{A}, \mathbf{B}$ ). We speculated that the causal mutation was filtered out due to low read coverage and/or quality. Thus we re-evaluated the candidate region (Chr7: 2-4 Mb) for each mutant and corrected the base quality and depth for sites of base quality $<20$ or coverage depth between 5 and 15 , based on GC content.

The candidate region was split into non-overlapping bins of $200 \mathrm{bp}$, and these bins were then grouped based on their GC contents (Figure 4A). For reference, $\sim 7,000$ bins of $200 \mathrm{bp}$ were randomly picked as background bins from other sequences of the same mutant and grouped according to their GC contents (Figure 4B). Total reads and total mapping quality were respectively calculated for each GC content group. The maximal base depth was set to 300 to minify the possible influences induced by repetitive regions. It is clear that, in both the candidate region and background bins, GC-rich and GC-poor bins have low read coverage as well as low mapping quality (Figures 4A,B).

The average mapping quality for bins in each GC content group was strongly correlated between the candidate region and background bins (Figure 4C), and so was the average read coverage (Figure 4D). Therefore, the bigger value of average quality or average read depth of the candidate region and background bins was chosen for calculation of quality coefficient or depth coefficient. Corrected quality or corrected depth was obtained by multiplying the quality coefficient or depth coefficient with the actual base quality or actual base depth for the site to be corrected, respectively. Sites with corrected quality or depth were then utilized for SNP calling and re-calculation of SNP index and $\mathrm{ED}^{6}$ values, resulting in 34 new SNPs in the candidate region. Five of them exhibited high SNP index and $\mathrm{ED}^{6}$ 
A

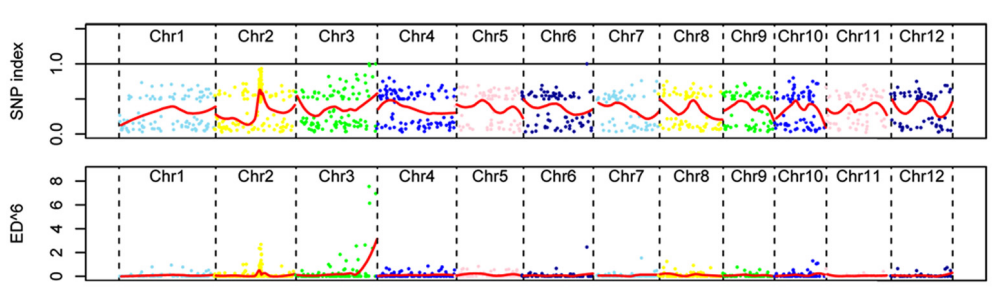

B

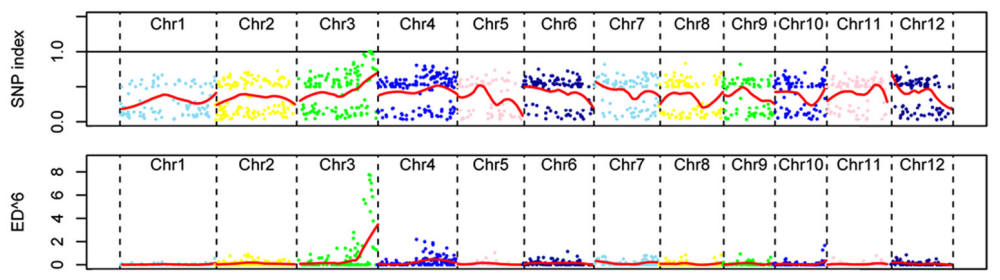

C

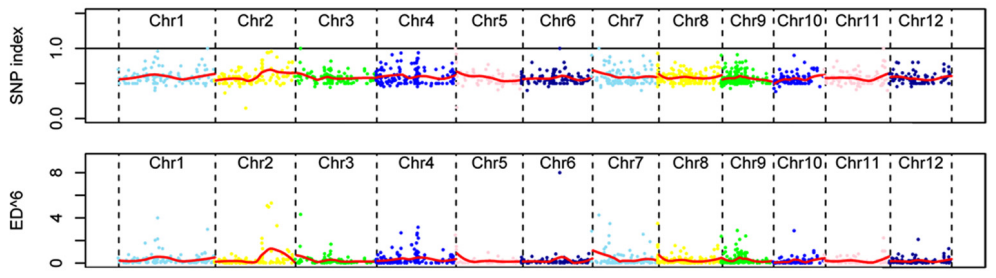

D
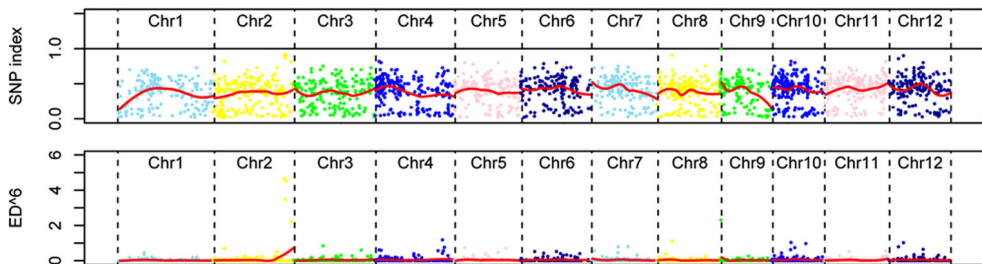

E
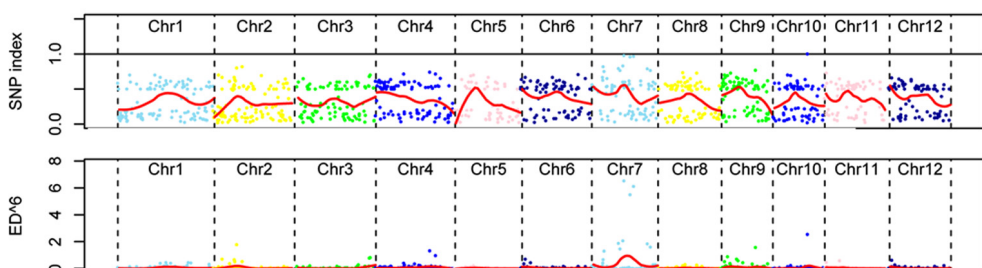

F
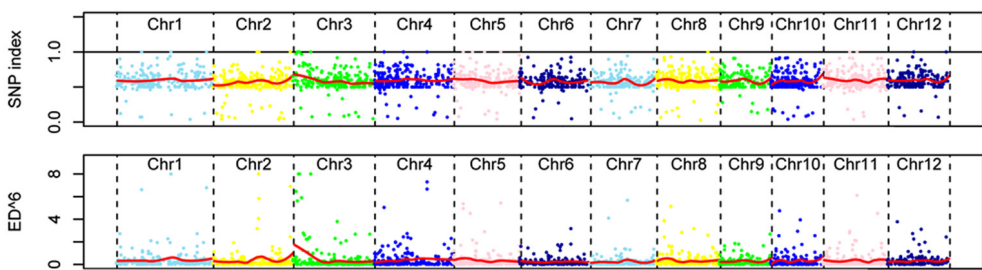

G

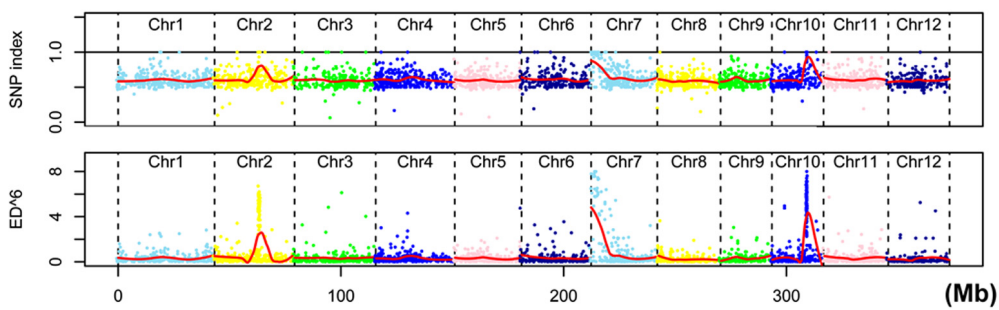

H-190

H-174

HM2-S55

HM2-S125

HM2-S61

HE-47

H-212

(Mb)

FIGURE 3 | Comparative plots of SNP indices and ED $^{6}$ values for seven HHZ mutants. (A) Mutant H-224. (B) Mutant HT5763. (C) Mutant H-190. (D) Mutant H-174. (E) Mutant HM2-S61. (F) Mutant HE-47. (G) Mutant H-212. Red lines in all plots represent Loess curve. 

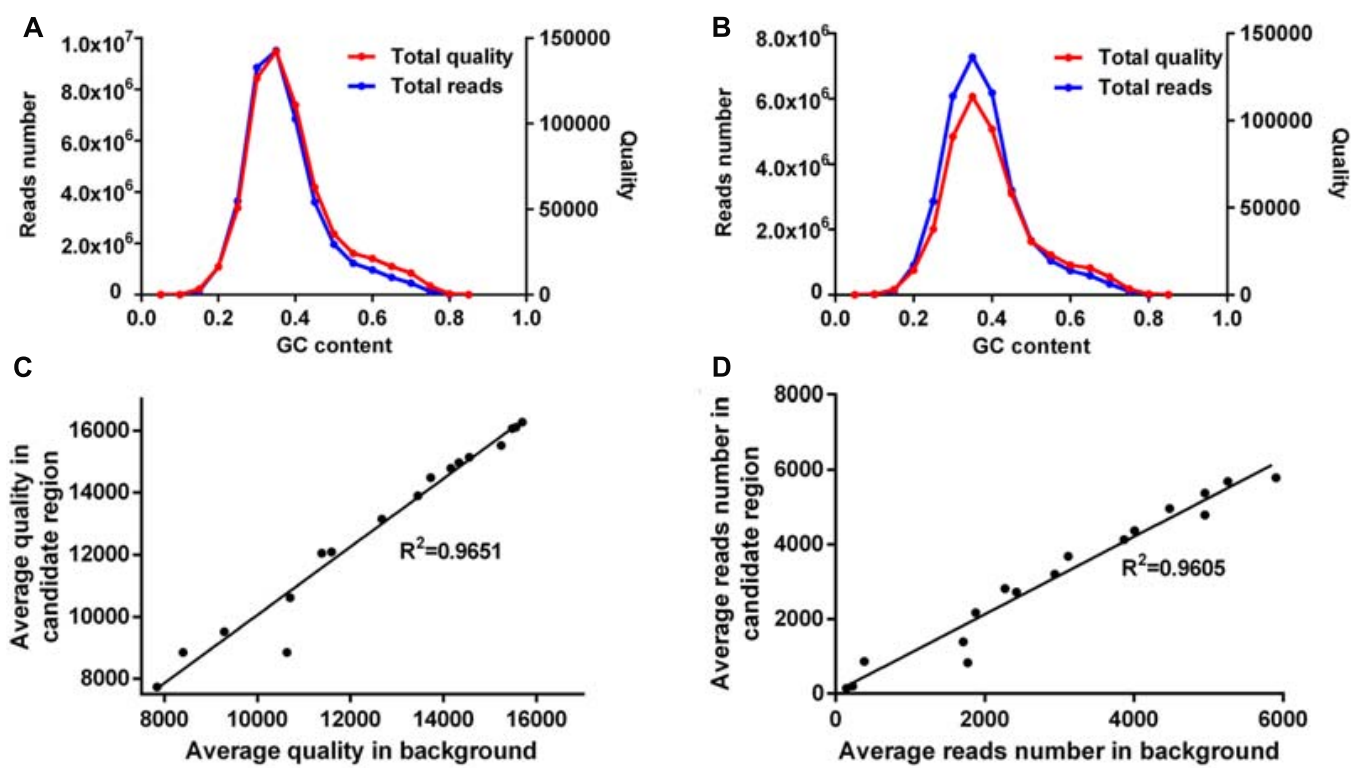

FIGURE 4 | Site corrections for mutant H-212. (A) The total reads number (left $y$-axis) and total mapping quality (right $y$-axis) within the candidate region (Chr7: 2 4 Mbp) are calculated for non-overlapping bins (200 bp) in each GC content group (increment 0.05). (B) Approximately 7,000 randomly selected bins from the whole genome are grouped based on their GC content. The total reads number (left $y$-axis) and total mapping quality (right $y$-axis) are calculated for bins in each GC content group (increment 0.05). The average quality (C) and average reads number (D) for bins in each GC content group are significantly correlated between the candidate region and background bins. Pearson's correlation $\left(R^{2}\right)$ is calculated for each comparison.

scores. One SNP located at Chr7: 2,068,093 induced a premature stop codon in LOC_Os07g04670 (G1) (Table 2). G1 encodes a transcriptional regulator. Yoshida et al. (2009) reported a G1 mutant allele of long sterile lemma phenotype consistent with what we observed for mutant $\mathrm{H}-212$.

\section{Phenotype Association Assay of the Candidate Genes}

Phenotype association assay was conducted to validate the candidate genes in seven HHZ mutants via HRM analysis (Lochlainn et al., 2011) using primers in Table 3. As expected, all the individuals of mutant phenotype carried a homozygous mutation. Approximately two-thirds of the plants with a wildtype phenotype were heterozygous for the mutation, and onethird were homozygous for the wild-type genotype (Table 3). The mutations in our candidate genes are thus very tightly associated with the mutant phenotype. Because the phenotypes we observed in our mutants were consistent with what in the published studies, we propose that SIMM correctly identified the causal mutations for the seven HHZ mutants.

\section{DISCUSSION}

The identification of causal mutations based on NGST provides an alternative approach for cloning of plant genes. Here we report a method that can simultaneously identify causal mutations in multiple mutants derived from the same parental plant. Different from the published methods such as MutMap (Abe et al., 2012) and NIKS (Nordstrom et al., 2013), SIMM does not need the sequence of the wild-type parent as reference, nor the assembly of sample specific $k$-mers. Instead, SIMM compares the test mutant with all other mutants as reference. This comparison scheme compensates for the sequencing deficiency in some mutant strains and avoids wrongly retaining or excluding candidate sites. As a result, SIMM can accurately resolve the causal mutation to many fewer candidate sites. The advantage of SIMM over MutMap and NIKS is shown by the analysis of seven published mutants in Table 1. For these mutants, the candidate sites resolved by SIMM were fewer but more complete than those resolved by MutMap or NIKS. Six candidate genes identified by SIMM were missed by MutMap, and two potentially valuable candidates were missed by NIKS as well (Table 1), In addition, MutMap yielded too many candidate sites for HHZ mutants, making it difficult to pinpoint the causal mutation. These results indicated that SIMM is more proficient than MutMap and NIKS in resolving candidate genes.

Two measures were included in the SIMM protocol to enhance accurate identification of causal mutations. First, all mutants were referenced for the identification of EMS-induced strain-specific SNPs. This is of key importance for SIMM to differentiate between causal mutations and background polymorphisms. Background polymorphisms between the test mutant and the wild-type parent are common, especially if the plants are derived from a recently established variety that still has many chromosomal segments heterogeneous in the population. Such background polymorphisms cannot be efficiently eliminated in pair-wise comparison between the mutant and wild-type genomes (as done in MutMap and 
TABLE 3 | Validation of causal mutations identified in $\mathrm{HHZ}$ mutants via co-segregation assay.

\begin{tabular}{|c|c|c|c|c|c|c|c|c|}
\hline \multirow[t]{2}{*}{ Sample } & \multirow[t]{2}{*}{ Phenotype } & \multirow[t]{2}{*}{ WT/Mutant } & \multirow[t]{2}{*}{$\chi^{2}(3: 1)$} & \multirow[t]{2}{*}{ Candidate gene } & \multirow[t]{2}{*}{ Primers } & \multicolumn{2}{|c|}{ WT } & \multirow{2}{*}{$\begin{array}{l}\text { Mutant } \\
\text { Homo }\end{array}$} \\
\hline & & & & & & Homo & Hetero & \\
\hline $\mathrm{H}-224$ & $\begin{array}{l}\text { Open hull and } \\
\text { brownish } \\
\text { palea/lemma }\end{array}$ & 299:85 & $0.0188(S)$ & LOC_Os03g43670 & $\begin{array}{l}\text { F: 5'-CAGCTGGTTCTGTGTTCAATTGTGGC-3' } \\
\text { R: 5'-CCTGCTCCTATAGACTGGAACCGCAC-3' }\end{array}$ & 27 & 63 & 26 \\
\hline HT5763 & Male sterile & $232: 80$ & $0.0008(\mathrm{~S})$ & LOC_Os04g39470 & $\begin{array}{l}\text { F: 5'-CCACTCGTTGATGCCTACCTGTTGC-3' } \\
\text { R: 5'-GGCTGCGGTGGACCAACTACCTC-3' }\end{array}$ & 39 & 72 & 30 \\
\hline $\mathrm{H}-190$ & Male sterile & $512: 264$ & $0.1444(\mathrm{NS})$ & LOC_Os03g58600 & $\begin{array}{l}\text { F: 5'-CATGTAGGTTGTGGCATC-3' } \\
\text { R: 5'-CCTGTCTATGTGGTTGAGC-3' }\end{array}$ & 42 & 76 & 35 \\
\hline $\mathrm{H}-174$ & Male sterile & $217: 67$ & $0.0043(\mathrm{~S})$ & LOC_Os03g58600 & $\begin{array}{l}\text { F: 5'-TGTGCTCTTGACATTCCT-3' } \\
\text { R: 5'-GCAAAGATTGTTGGTCAG-3' }\end{array}$ & 21 & 58 & 25 \\
\hline HM2-S61 & Male sterile & $251: 93$ & $0.0083(\mathrm{~S})$ & LOC_Os07g32480 & $\begin{array}{l}\text { F: 5'-GCACCAAGGTGGTAGAAAG-3' } \\
\text { R: 5'-AGCAACCAGGTCTTCTGT-3' }\end{array}$ & 24 & 55 & 32 \\
\hline $\mathrm{HE}-47$ & $\begin{array}{l}\text { Red lesions on } \\
\text { leaves }\end{array}$ & $97: 39$ & $0.0257(S)$ & LOC_Os03g06410 & $\begin{array}{l}\text { F: 5'-TGGAGTTGAATAGAGTGT-3' } \\
\text { R: 5'-GAATATCCCTCGTITACT-3' }\end{array}$ & 36 & 90 & 42 \\
\hline $\mathrm{H}-212$ & $\begin{array}{l}\text { Long sterile } \\
\text { lemma }\end{array}$ & $132: 44$ & $0.0000(S)$ & LOC_Os07g04670 & $\begin{array}{l}\text { F: 5'-TGCTCGCCGGCGGAGCTG-3' } \\
\text { R: 5'-GCGAGGTACTGCGTGAAG-3' }\end{array}$ & 30 & 85 & 45 \\
\hline
\end{tabular}

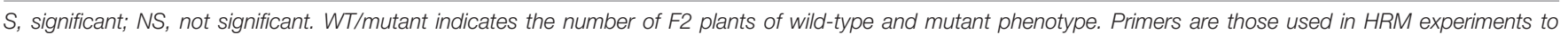

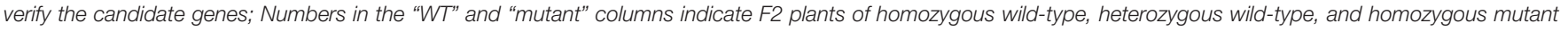
genotypes shown by HRM experiment.

NIKS), but can be eliminated or significantly reduced when multiple mutants are compared. SIMM cuts down the number of strain-specific SNPs to $6 \sim 70 \%$ of those identified using MutMap (Table 2, \#SNPs ${ }^{2}$ vs. \#SNPs ${ }^{3}$ ). Thereby, a large number of background polymorphisms were excluded for subsequent analysis. Second, AI and ED analyses were introduced into the SIMM algorithm, which significantly reduced the background noises caused by sequencing deficiencies, and increased the reliability of genotyping the wild-type and mutant alleles for the test mutant. The application of AI and ED analyses reduced not only the number of candidate SNPs to be experimentally verified, but also the chance to wrongly exclude the causal mutations.

Sequences generated by the Illumina Hiseq platform usually tend to have low read coverage in GC-rich or AT-rich regions (Dohm et al., 2008; Benjamini and Speed, 2012). We also found low mapping quality for these regions. If the causal mutation happens to be in such a region, it would be filtered out by the cutoff criteria for coverage depth and sequence quality. This is exemplified by mutant $\mathrm{H}-212$, in which a candidate region was correctly identified but the causal mutation was not found. Further examination of reads covering the candidate region revealed the presence of sequence bias. Corrections of read quality and coverage depth were performed to the sites filtered out due to the sequence bias, based on the GC content. Using the corrected quality and depth for SNP index and ED analyses, a potential causal mutation site was identified for mutant $\mathrm{H}-212$.

The SIMM method is particularly suitable when multiple mutants derived from the same parental plant are to be analyzed. We conducted a statistical analysis to assess the influence of the number of background mutants on the power of SIMM to resolve the strain-specific SNPs, based on the seven HHZ mutants. As shown in Figure 5, the strain-specific SNPs reduced dramatically when 3-5 background mutants were used. However, the resolution power did not increase significantly

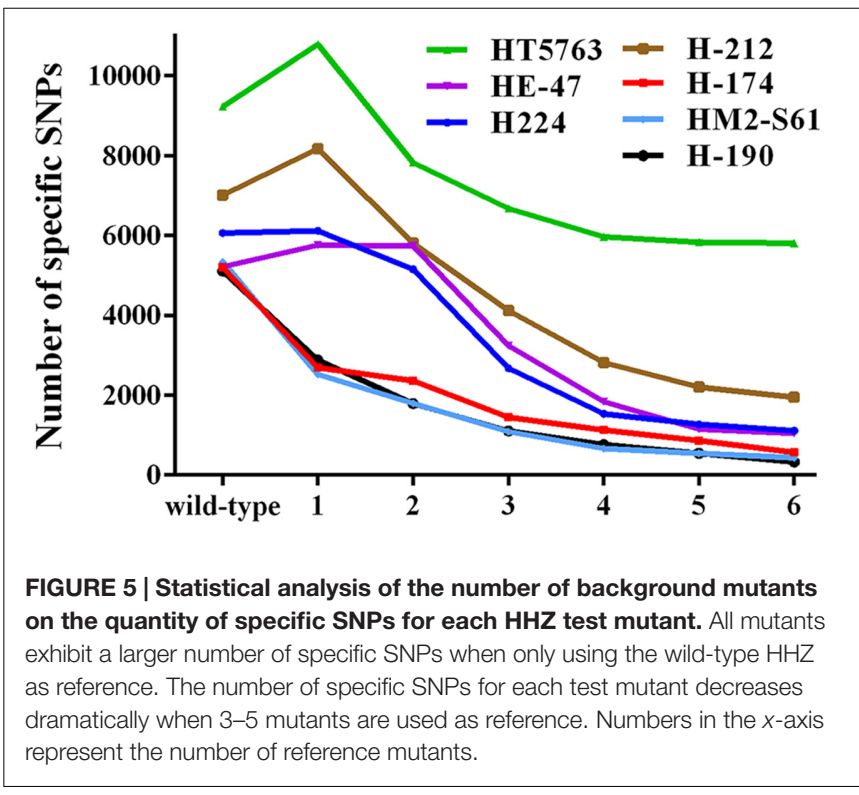

when five or more mutants were analyzed. Thus, sequencing 35 mutants is suggested for SIMM when dealing with mutants of less background polymorphisms. However, sequencing of more mutants is necessary if they have higher background polymorphisms. When only one or two mutants are available, then bulk sequencing of a few wild-type plants derived from the same parental line is recommended. The presence of a sibling mutant derived from the same mutant line can wipe out candidate regions for both siblings. However, such mutants can be recognized by the SIMM pipeline, which then analyzes them individually. Identical mutant alleles derived from independent mutagenesis events show overlapping candidate regions, and the SIMM pipeline can recognize such mutants and process them separately. 


\section{CONCLUSION}

Simultaneous identification of multiple mutations is a proficient method that can simultaneously resolve the causal genes in multiple mutants without the need to sequence the wild-type genome. By introducing AI analysis, ED analysis, and site correction, SIMM can accurately resolve the causal mutation to one or a few candidate sites. SIMM showed better sensitivity and specificity than MutMap and NIKS when analyzing seven published rice mutants. Applications on seven HHZ mutants further showed the efficacy of SIMM for NGST-based identification of mutant plant genes.

\section{ACCESSION NUMBERS}

Sequencing data can be found in the NCBI Sequence Read Archive under accession number SRP058039.

\section{AUTHOR CONTRIBUTIONS}

$\mathrm{XD}, \mathrm{HH}$, and $\mathrm{XT}$ conceived the ideas for the study; WY and $\mathrm{HH}$ designed the algorithm; ZC, JL, CX, GX, and YL performed

\section{REFERENCES}

Abe, A., Kosugi, S., Yoshida, K., Natsume, S., Takagi, H., Kanzaki, H., et al. (2012). Genome sequencing reveals agronomically important loci in rice using MutMap. Nat. Biotechnol. 30, 174-178. doi: 10.1038/nbt. 2095

Allen, G. C., Flores-Vergara, M. A., Krasynanski, S., Kumar, S., and Thompson, W. F. (2006). A modified protocol for rapid DNA isolation from plant tissues using cetyltrimethylammonium bromide. Nat. Protoc. 1, 2320-2325. doi: 10. 1038/nprot.2006.384

Allen, R. S., Nakasugi, K., Doran, R. L., Millar, A. A., and Waterhouse, P. M. (2013). Facile mutant identification via a single parental backcross method and application of whole genome sequencing based mapping pipelines. Front. Plant Sci. 4:362. doi: 10.3389/fpls.2013.00362

Austin, R. S., Vidaurre, D., Stamatiou, G., Breit, R., Provart, N. J., Bonetta, D., et al. (2011). Next-generation mapping of Arabidopsis genes. Plant J. 67, 715-725. doi: 10.1111/j.1365-313X.2011.04619.x

Benjamini, Y., and Speed, T. P. (2012). Summarizing and correcting the GC content bias in high-throughput sequencing. Nucleic Acids Res. 40:e72. doi: 10.1093/nar/ gks001

Dohm, J. C., Lottaz, C., Borodina, T., and Himmelbauer, H. (2008). Substantial biases in ultra-short read data sets from high-throughput DNA sequencing. Nucleic Acids Res. 36:e105. doi: 10.1093/nar/gkn425

Fatland, B. L., Nikolau, B. J., and Wurtele, E. S. (2005). Reverse genetic characterization of cytosolic acetyl-CoA generation by ATP-citrate lyase in Arabidopsis. Plant Cell 17, 182-203. doi: 10.1105/tpc.104.026211

Fekih, R., Takagi, H., Tamiru, M., Abe, A., Natsume, S., Yaegashi, H., et al. (2013). MutMap+: genetic mapping and mutant identification without crossing in rice. PLoS ONE 8:e68529. doi: 10.1371/journal.pone.0068529.

Higginson, T., Li, S. F., and Parish, R. W. (2003). AtMYB103 regulates tapetum and trichome development in Arabidopsis thaliana. Plant J. 35, 177-192. doi: 10.1046/j.1365-313X.2003.01791.x

Hill, J. T., Demarest, B. L., Bisgrove, B. W., Gorsi, B., Su, Y. C., and Yost, H. J. (2013). MMAPPR: mutation mapping analysis pipeline for pooled RNA-seq. Genome Res. 23, 687-697. doi: 10.1101/gr.146936.112

Jacoby, W. G. (2000). Loess: a nonparametric, graphical tool for depicting relationships between variables. Elect. Stud. 19, 577-613. doi: 10.1016/S02613794(99)00028-1 the experiments; all the authors participated in data analysis and interpretation, and contributed to the writing and editing of the manuscript.

\section{FUNDING}

This work was supported by funds from Guangdong Innovative Research Team Program [201001S0104725509]; the Ministry of Agriculture Transgenic Project [2012ZX08001001]; the National Program on Key Basic Research Project of China [973 Program: 2011CB100101, 2013CBA01402]; the National High Technology Research and Development Program of China [863 Program: 2014AA10A602]; Natural Science Foundation of China [31110103917]; Shenzhen Commission on Innovation and Technology [KQF201109160004A, CXZZ20140411140647863].

\section{ACKNOWLEDGMENTS}

We are grateful to Yong Qiang Gu and Toni Mohr for critical reading and editing of the paper. We thank Zhenyi Chang and Dongfeng Liu for assistance in HRM analysis.

Jander, G., Norris, S. R., Rounsley, S. D., Bush, D. F., Levin, I. M., and Last, R. L. (2002). Arabidopsis map-based cloning in the post-genome era. Plant Physiol. 129, 440-450. doi: 10.1104/pp.003533

Langmead, B., and Salzberg, S. L. (2012). Fast gapped-read alignment with Bowtie 2. Nat. Methods 9, 357-359. doi: 10.1038/nmeth.1923

Li, H., and Durbin, R. (2009). Fast and accurate short read alignment with BurrowsWheeler transform. Bioinformatics 25, 1754-1760. doi: 10.1093/bioinformatics/ btp324

Li, H., Handsaker, B., Wysoker, A., Fennell, T., Ruan, J., Homer, N., et al. (2009). The sequence alignment/map format and SAMtools. Bioinformatics 25, 20782079. doi: 10.1093/bioinformatics/btp352

Li, R., Li, Y., Fang, X., Yang, H., Wang, J., Kristiansen, K., et al. (2009a). SNP detection for massively parallel whole-genome resequencing. Genome Res. 19, 1124-1132. doi: 10.1101/gr.088013.108

Li, R., Yu, C., Li, Y., Lam, T. W., Yiu, S. M., Kristiansen, K., et al. (2009b). SOAP2: an improved ultrafast tool for short read alignment. Bioinformatics 25, 1966-1967. doi: 10.1093/bioinformatics/btp336

Lochlainn, S. O., Amoah, S., Graham, N. S., Alamer, K., Rios, J. J., Kurup, S., et al. (2011). High Resolution Melt (HRM) analysis is an efficient tool to genotype EMS mutants in complex crop genomes. Plant Methods 7:43. doi: 10.1186/17464811-7-43

Lukowitz, W., Gillmor, C. S., and Scheible, W. R. (2000). Positional cloning in Arabidopsis. Why it feels good to have a genome initiative working for you. Plant Physiol. 123, 795-805. doi: 10.1104/pp.123.3.795

Martin, M. (2011). Cutadapt removes adapter sequences from high-throughput sequencing reads. Embnet J. 17, 10-12. doi: 10.14806/ej.17.1.200

Nonomura, K., Morohoshi, A., Nakano, M., Eiguchi, M., Miyao, A., Hirochika, H., et al. (2007). A germ cell specific gene of the ARGONAUTE family is essential for the progression of premeiotic mitosis and meiosis during sporogenesis in rice. Plant Cell 19, 2583-2594. doi: 10.1105/tpc.107. 053199

Nordstrom, K. J., Albani, M. C., James, G. V., Gutjahr, C., Hartwig, B., Turck, F., et al. (2013). Mutation identification by direct comparison of whole-genome sequencing data from mutant and wild-type individuals using $k$-mers. Nat. Biotechnol. 31, 325-330. doi: 10.1038/nbt.2515

Peters, J. L., Cnudde, F., and Gerats, T. (2003). Forward genetics and map-based cloning approaches. Trends Plant Sci. 8, 484-491. doi: 10.1016/j.tplants.2003. 09.002 
Phan, H. A., Iacuone, S., Li, S. F., and Parish, R. W. (2011). The MYB80 transcription factor is required for pollen development and the regulation of tapetal programmed cell death in Arabidopsis thaliana. Plant Cell 23, 22092224. doi: 10.1105/tpc.110.082651

Phan, H. A., Li, S. F., and Parish, R. W. (2012). MYB80, a regulator of tapetal and pollen development, is functionally conserved in crops. Plant Mol. Biol. 78, 171-183. doi: 10.1007/s11103-011-9855-0.

Pu, C. X., Ma, Y., Wang, J., Zhang, Y. C., Jiao, X. W., Hu, Y. H., et al. (2012). Crinkly4 receptor-like kinase is required to maintain the interlocking of the palea and lemma, and fertility in rice, by promoting epidermal cell differentiation. Plant J. 70, 940-953. doi: 10.1111/j.1365-313X.2012. 04925.x

Schneeberger, K., Ossowski, S., Lanz, C., Juul, T., Petersen, A. H., Nielsen, K. L., et al. (2009). SHOREmap: simultaneous mapping and mutation identification by deep sequencing. Nat. Methods 6, 550-551. doi: 10.1038/nmeth0809-550

Shen, X., Liu, H., Yuan, B., Li, X., Xu, C., and Wang, S. (2011). OsEDR1 negatively regulates rice bacterial resistance via activation of ethylene biosynthesis. Plant Cell Environ. 34, 179-191. doi: 10.1111/j.1365-3040.2010.02219.x

Takagi, H., Uemura, A., Yaegashi, H., Tamiru, M., Abe, A., Mitsuoka, C., et al. (2013). MutMap-Gap: whole-genome resequencing of mutant F2 progeny bulk combined with de novo assembly of gap regions identifies the rice blast resistance gene Pii. New Phytol. 200, 276-283. doi: 10.1111/nph. 12369

Uchida, N., Sakamoto, T., Kurata, T., and Tasaka, M. (2011). Identification of EMSinduced causal mutations in a non-reference Arabidopsis thaliana accession by whole genome sequencing. Plant Cell Physiol. 52, 716-722. doi: 10.1093/pcp/ pcr029

Wang, M., Tang, D., Luo, Q., Jin, Y., Shen, Y., Wang, K., et al. (2012). BRK1, a Bub1related kinase, is essential for generating proper tension between homologous kinetochores at metaphase I of rice meiosis. Plant Cell 24, 4961-4973. doi: 10.1105/tpc.112.105874

Yoshida, A., Suzaki, T., Tanaka, W., and Hirano, H. Y. (2009). The homeotic gene long sterile lemma (G1) specifies sterile lemma identity in the rice spikelet. Proc. Natl. Acad. Sci. U.S.A. 106, 20103-20108. doi: 10.1073/pnas.0907896106

Zhu, Y., Mang, H. G., Sun, Q., Qian, J., Hipps, A., and Hua, J. (2012). Gene discovery using mutagen-induced polymorphisms and deep sequencing: application to plant disease resistance. Genetics 192, 139-146. doi: 10.1534/ genetics.112.141986

Conflict of Interest Statement: The authors declare that the research was conducted in the absence of any commercial or financial relationships that could be construed as a potential conflict of interest.

Copyright (c) 2017 Yan, Chen, Lu, Xu, Xie, Li, Deng, He and Tang. This is an openaccess article distributed under the terms of the Creative Commons Attribution License (CC BY). The use, distribution or reproduction in other forums is permitted, provided the original author(s) or licensor are credited and that the original publication in this journal is cited, in accordance with accepted academic practice. No use, distribution or reproduction is permitted which does not comply with these terms. 